www.jmscr.igmpublication.org

Impact Factor 5.84

Index Copernicus Value: 71.58

ISSN (e)-2347-176x ISSN (p) 2455-0450

crossref DOI: _https://dx.doi.org/10.18535/jmscr/v5i11.210

Journal Of Medical Science And Clinical Research

IGM Publication

An Official Publication of IGM Publication

\title{
Psychosocial Evalution for Prevalence of Sub-Types of Somatoform Disorders in Diagnosed Patients of Somatoform Disorders Visiting Psychosomatic Clinic in Tertiary Care Centre, Vidarbha
}

\author{
Authors \\ Ratika Sharma ${ }^{1}$, Prakriti Vohra ${ }^{1^{*}}$ \\ ${ }^{1}$ Senior Resident, Deptt. of Psychiatry, SHKM, GMC, Mewat \\ ${ }^{1 *}$ Assistant Professor, Deptt. of Microbiology, SHKM, GMC, Mewat \\ Corresponding Author ${ }^{1 *}$ \\ Dr Prakriti Vohra \\ Assistant Professor, SHKM, GMC, Mewat, Haryana, 122107 \\ Email:Prakriti.doc@gmail.com, Contact number: 9992289955
}

\begin{abstract}
Introduction: Somatoform disorders are a group of psychiatric disorders in which patients present with clinically significant but unexplained physical symptoms. The disorders include somatization disorder, undifferentiated somatoform disorder, hypochondriasis, conversion disorder, pain disorder, body dysmorphic disorder, and somatoform disorder not otherwise specified.

Material and Methods: 270 patients with a diagnosis of any of the sub types of somatoform disorders were inducted from those attending the out-patient and in-patient services of Department. Of Psychiatry affiliated to AVBRH, a tertiary care hospital, Vidarbha. It was a cross-sectional study carried out from October 2010 to July 2012. The socio-demographic profile questionnaire, clinical profile sheet and the Bradford somatoform inventory (BSI) for diagnosing somatoform disorder patients was used.

Results: various subtypes of somatoform disorders were diagnosed.

Conclusion: Various sub types of somatoform disorders along with different duration have emerged.

Keywords: Somatoform disorders, sub-types of somatoform disorders, prevalence.
\end{abstract}

\section{Introduction}

Somatoform disorders are a group of psychiatric disorders in which patients present with clinically significant but unexplained physical symptoms. The disorders include somatization disorder, undifferentiated somatoform disorder, hypochondriasis, conversion disorder, pain disorder, body dysmorphic disorder, and somatoform disorder not otherwise specified. ${ }^{1}$ These disorders often cause significant emotional distress for patients and are a challenge to physicians. Somatic symptoms account for more than half of all ambulatory visits or nearly 400 million clinic visits in the United States alone each year. ${ }^{2} \mathrm{~A}$ survey in 3000 primary care patients revealed prevalence's ranging from $23 \%$ to $63 \%$ for 12 of the symptoms. ${ }^{3}$ The reported prevalence of the disorder has varied with $0.1 \%$ in the general population and the prevalence increase to $11.6 \%$ (Yates) when diagnostic criteria are not properly applied. Fink et $a l^{4}$ reported a prevalence of somatoform disorders as high as $30.3 \%$ whereas 
Pehlivanturk B et al ${ }^{5}$ reported the prevalence of somatization disorder as $2 \%$. A study in Belgium reported that somatization syndrome is the third highest psychiatric disorder, with a prevalence rate of $8.9 \%$. The first and second most common psychiatric disorders were depression and anxiety disorder. $^{6}$ Patients with somatization disorder usually present with numerous symptoms. ${ }^{7}$ Somatic symptoms, especially vegetative symptoms such as changes in appetite or weight, lack of pleasure and sexual appetence, sleep abnormalities; dysfunctional hypothalamic and sleep centers may be of paramount importance as all of them are influenced by both serotonin and nor epinephrine. ${ }^{8}$ The specific criteria for diagnosis of this disorder are given in the DSM-IV and in ICD -10. DSM-IV lists only three criteria of somatic symptoms for major depressive disorder: sleep disturbance, appetite disturbance, and fatigue or loss of energy and correspondingly in ICD-10, disturbances of sleep and appetite, loss of libido, and amenorrhea are the only somatic symptoms considered to be of diagnostic significance for major depression.

\section{Material and Methods}

The patients were inducted from those attending the out-patient and in-patient services of Department of Psychiatry affiliated to AVBRH a tertiary care hospital, Sawangi, (Meghe), D.M.I.M.S University. Study was commenced only after approval from Institutional Ethical Committee. Informed Consent of the patient and their care giver was duly obtained before their evaluation. The sample consisted of 270 patients with a diagnosis of any of the sub types of somatoform disorders. It was a cross-sectional, naturalistic pilot study with single time assessment.

The study was carried out from October 2010 to July 2012. Statistical assessment using descriptive and analytical methods was done appropriately after the collection of data.
Bradford Somatoform Inventory (BSI): It was developed by the British Neuropsychologist Mumford and Bavington in 1991 in the University Of Bradford, U.K. ${ }^{9}$ The inventory was constructed simultaneously in Urdu and English by recruiting multi-Ethnic populations of Pakistani origin as well as indigenous British patients with a clinical diagnosis of depression, anxiety, hysteria and hypochondriasis. Further the pilot version of the somatic inventory was checked against psychiatric case notes in North and South India as well as in Nepal. The final revised version of the inventory had total of 45 items to be recorded on 3 point Likert scale, with the demarcating point of presence of symptoms for less than or more than 15 days in the last month in the sufferer. The linguistic and conceptual equivalence had been fairly established and explored using factor analysis with four principal factors (head, chest, abdomen and fatigue). It had adequate inter-rater reliability (kappa, $\mathrm{k}=0.87$ ) as well as adequate face and content. Validity, (it achieved over $90 \%$ coverage of all somatic symptoms recorded in each centre during its development).

\section{Results}

Our study showed diagnostic distribution of various subtypes of somatoform disorders. $40.7 \%$ were diagnosed with somatization disorder and there were two sub groups of somatoform disorder viz. somatoform autonomic nervous system disorder and somatoform disorder not otherwise specified, both accounting to almost similar distribution( $n=4,14.8 \%)$. 
The results of diagnostic distribution are as follows

\section{Diagnostic distribution}

Table 1: Distribution of somatoform disorder

\begin{tabular}{|l|c|c|c|c|c|}
\hline \multirow{2}{*}{ Diagnosis } & \multicolumn{2}{|c|}{ Sex } & & \multicolumn{2}{c|}{ N (\%) } \\
\cline { 2 - 6 } & $\begin{array}{c}\text { Male } \\
\text { N (\%) }\end{array}$ & $\begin{array}{c}\text { Female } \\
\mathbf{N}(\%)\end{array}$ & Total & Spontaneous visit & $\begin{array}{c}\text { Referred from } \\
\text { other dept }\end{array}$ \\
\hline F45.0 & $42(15.56 \%)$ & $68(25.19 \%)$ & $110(40.74 \%)$ & $73(66.36 \%)$ & $37(33.63 \%)$ \\
\hline F45.1 & $15(5.56 \%$ & $18(6.67 \%)$ & $33(12.22 \%)$ & $21(66.33 \%)$ & $12(36.36 \%)$ \\
\hline F45.2 & $5(1.85 \%)$ & $10(3.70 \%)$ & $15(5.56 \%)$ & $11(73.33 \%)$ & $4(26.66 \%)$ \\
\hline F45.3 & $18(6.67 \%)$ & $22(8.15 \%)$ & $40(14.81 \%)$ & $12(30 \%)$ & $28(70 \%)$ \\
\hline F45.4 & $13(4.81 \%)$ & $16(5.93 \%)$ & $29(10.74 \%)$ & $19(65.51 \%)$ & $10(34.48 \%)$ \\
\hline F45.8 & $15(5.56 \%)$ & $25(9.26 \%)$ & $40(14.81 \%)$ & $32(80 \%)$ & $8(20 \%)$ \\
\hline F45.9 & $1(0.37 \%)$ & $2(0.74 \%)$ & $3(1.11 \%)$ & $3(100 \%)$ & $0(0 \%)$ \\
\hline & $117(40.37 \%)$ & $153(59.63 \%)$ & 270 & $171(63.3 \%)$ & $99(36.67 \%)$ \\
\hline
\end{tabular}

$\mathrm{P}$ value-0.947; Chi-Square t test is applied. $\mathrm{P}$ value is significant if $<0.05$.

\section{Graph 1}

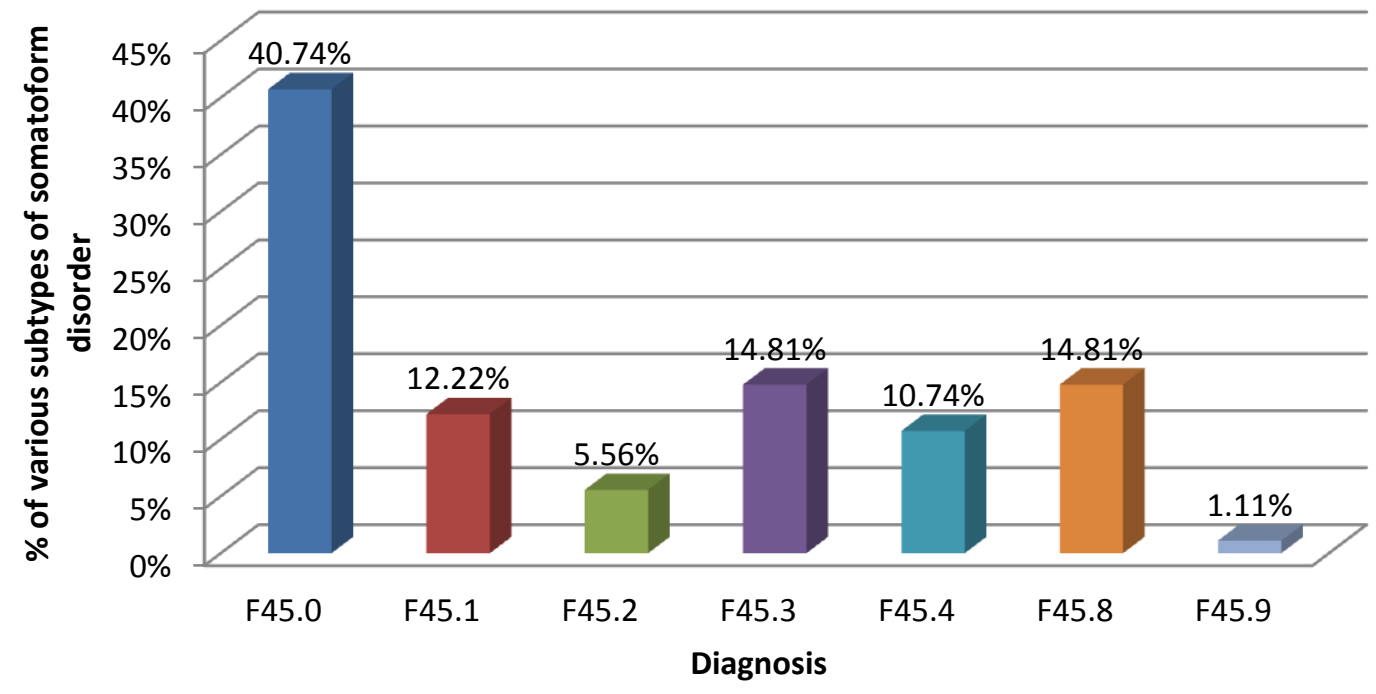

Table 1 shows diagnostic distribution of various subtypes of somatoform disorders. The diagnoses were made according to ICD-10 which predominantly shows $(\mathrm{n}=110), 40.7 \%$ were diagnosed with somatization disorder and there were two sub groups of somatoform disorder viz. somatoform autonomic nervous system disorder and somatoform disorder not otherwise specified, both accounting to almost similar distribution( $\mathrm{n}=$ 4, 14.8\%). Unspecified somatoform disorder. There was no statistical significant $(\mathrm{p}=0.947)$ difference discernable among the subtypes of somatoform disorder. As far as the referral was concerned we noticed majority of (63.3\%) 171 out of 270 patients coming spontaneously to psychiatric services and $36.67 \%$ (99 out of 270) of patients were referred from other departments of our tertiary care hospital. Most of the referrals were from the category of autonomic nervous system $(70 \%)$. Followed by undifferentiated and further followed by persistent somatoform pain disorder.

Table 2: Patterns of onset and duration of somatoform disorder

\begin{tabular}{|l|l|l|l|l|l|l|}
\hline Gender & $\mathrm{N}$ & Mean & Median & SD & Range & p-value \\
\hline Male & $117(43.33 \%)$ & 57.44 & 65 & 16.64 & $12-88$ & 0.15 \\
\cline { 1 - 5 } Female & $153(56.67 \%)$ & 54.73 & 60 & 14.36 & $15-80$ & NS,p $>0.05$ \\
\cline { 1 - 5 } Total & $270(100 \%)$ & 55.90 & 60 & 15.42 & $12-88$ & \\
\hline
\end{tabular}

Unpaired t test is applied. P value is significant if $<0.05$. 
Table 2-A: Pattern of course of illness

\begin{tabular}{|c|c|c|c|c|c|c|c|}
\hline \multirow{2}{*}{ Diagnosis } & \multirow{2}{*}{$\mathbf{N}$} & \multicolumn{4}{|c|}{ Course } & א2-value & p-value \\
\hline & & Episodic & $\%$ & Continuous & $\%$ & & \\
\hline F45.0 & 110 & 19 & $17.27 \%$ & 91 & $82.72 \%$ & & \\
\hline F45.1 & 33 & 9 & $27.27 \%$ & 24 & $72.72 \%$ & & \\
\hline F45.2 & 15 & 10 & $66.66 \%$ & 05 & $33.33 \%$ & & \\
\hline F45.3 & 40 & 15 & $37.50 \%$ & 25 & $62.50 \%$ & 3.00 & $\begin{array}{c}0.80 \\
N S n>0.05\end{array}$ \\
\hline F45.4 & 29 & 12 & $41.37 \%$ & 17 & $58.62 \%$ & & \\
\hline F45.8 & 40 & 8 & $20.00 \%$ & 32 & $80.00 \%$ & & \\
\hline F45.9 & 3 & 2 & $66.66 \%$ & 01 & $33.33 \%$ & & \\
\hline Total & 270 & 75 & $27.77 \%$ & 195 & $72.22 \%$ & & \\
\hline
\end{tabular}

\section{Graph 2A}

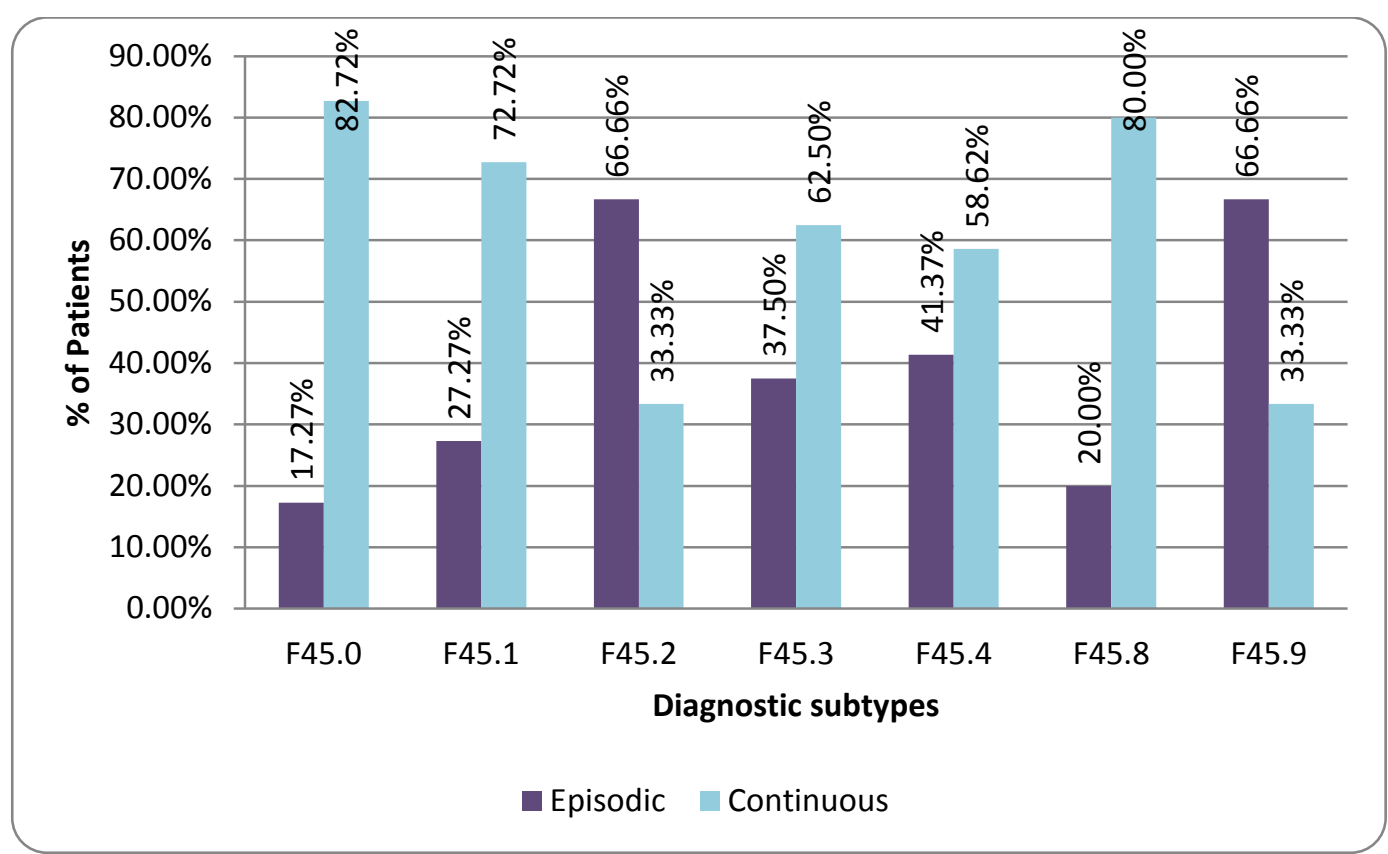

Table 2A - showing the course of illness in patients of somatoform disorder(s). A total of 75 disorders have clearly shown a continuous course (27.78\%) patients have shown an episodic course compared to $195(72.22 \%)$ patients who showed followed by $80 \%$ of somatoform disorder not otherwise specified following the same continuous pattern. continuous course. $82.72 \%$ of somatization

Table 2-B: Showing the mean and median duration of various subtypes of somatoform disorder

\begin{tabular}{|l|c|c|c|c|c|c|}
\hline Diagnosis & N & Mean & Median & SD & Range & p-value \\
\hline F45.0 & 110 & 10.2 & 11 & 2.31 & $2-14$ & \\
F45.1 & 33 & 2.31 & 4 & 1.21 & $1-5$ & \\
F45.2 & 15 & 11.21 & 12 & 3.42 & $3-15$ & \\
F45.3 & 40 & 13.12 & 13 & 4.12 & $4-12$ & 0.04 \\
F45.4 & 29 & 3.32 & 2 & 1.12 & $1-4$ & S,p $<0.05$ \\
F45.8 & 40 & 2.13 & 2 & 1.21 & $1-3$ & \\
F45.9 & 3 & 3.12 & 4 & 1.36 & $1-5$ & \\
Total & 270 & 6.36 & 13 & 2.16 & $1-15$ & \\
\hline
\end{tabular}

Unpaired t test is applied. $\mathrm{P}$ value is significant if $<0.05$. 


\section{Graph 2 B}

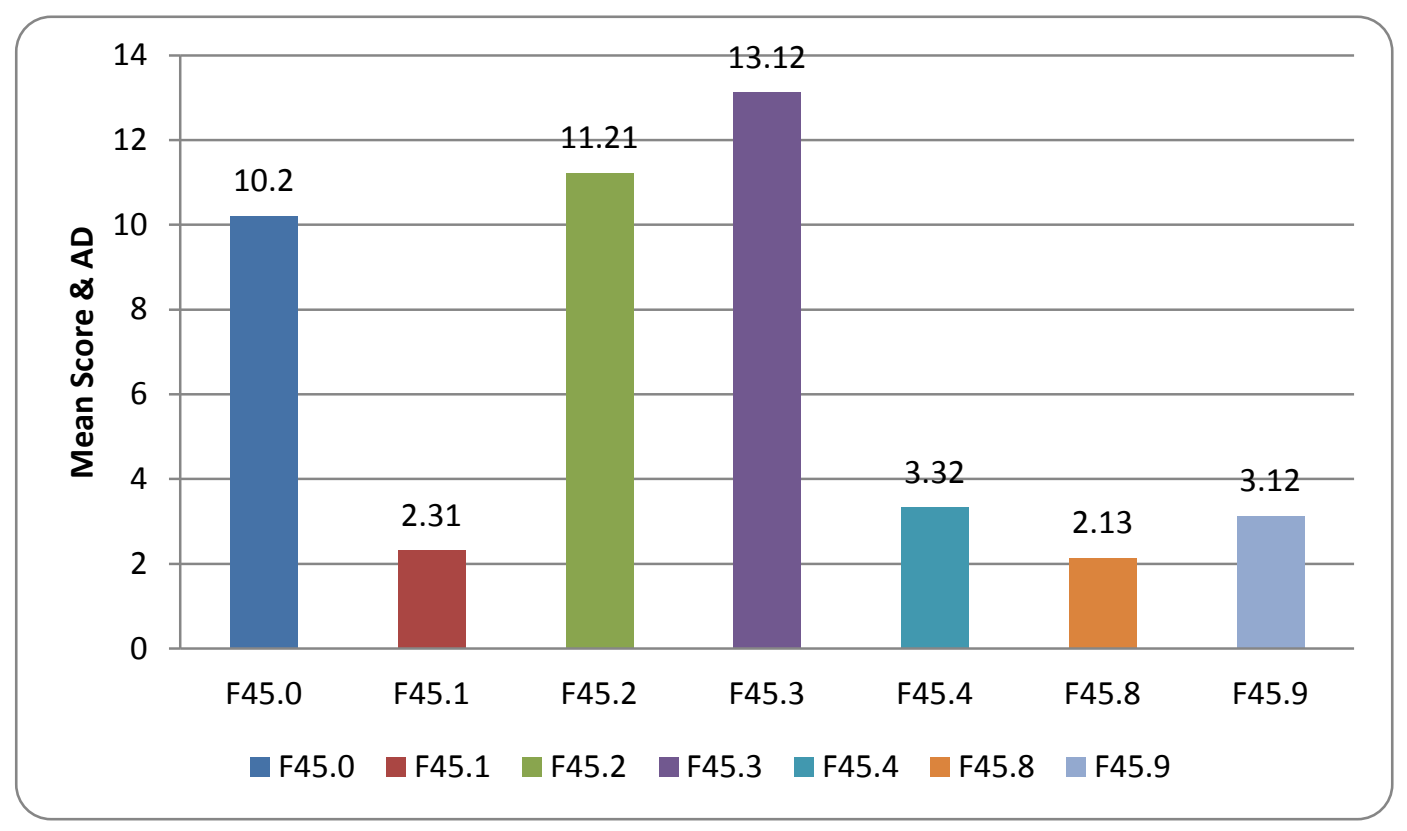

Table 2-B showing the mean and median duration of various subtypes of somatoform disorders.

Somatoform autonomic nervous system subtype has shown a mean of 13.12 years and has emerged as the longest lasting subtype from this part of the country. It was followed by hypochondriasis (mean 11.21 years, median is 12 years) further followed by somatization disorder $(10.2 \pm 2.31$ years), the median is 11 years. Somatoform disorder not otherwise specified has shown the shortest duration (2.13 years, a median of 2 years) among all the subtypes. The overall dispersion of duration is found to be statistically significant $(\mathrm{p}=0.04)$.

\section{Discussion}

The discussion aims to examine the prevalence of various types of somatoform disorder(s) viz, somatization disorder, undifferentiated somatoform disorder, hypochondriasis, conversion disorder, pain disorder, body dysmorphic disorder, and somatoform disorder not otherwise specified, in the broadly categorized and diagnosed patients of somatoform disorder(s), in relation to the result of the present study and its comparison with the previously published studies. Our search on MEDLINE and EMBASE did not show any study from India in this particular regard, hence, this study was planned in patients of somatoform disorder visiting psychosomatic clinic in tertiary care hospital, from Vidarbha region to evaluate the prevalence of sub types of somatoform disorders in total sample of 270 psychiatric outpatients who met the diagnostic criteria for broadly somatoform disorder(s). In our study majority of the patients had somatization disorder (40.7\%), followed by somatoform autonomic dysfunction and not otherwise specified somatoform disorder, each accounting to $14.8 \%$ respectively. In majority of patients $(72.22 \%$, $\mathrm{n}=195)$ had continuous course, whereas $(27.77 \%$, $\mathrm{n}=75$ ) had episodic course in our sample. Aigner et al in $2003^{10}$ have only taken 147 patients of persistent somatoform pain disorder when he studied for the prevalence of sub types of somatoform disorder(s), whereas Nakao et al ${ }^{11}$ who have evaluated 863 out patients on Cornell Medical Index Questionnaire and failed to mention the subtypes of somatoform disorder in his study for identifying the different patterns in them; and since our study is the first one in unique sense to identify and evaluate the prevalence of various sub types of somatoform disorders in patients of broadly catagorized somatoform disorder(s), it gives us clear focus when we discuss about subtypes of them. 


\section{Conclusions}

This study aimed to examine the prevalence of sub types of somatoform disorders in diagnosed patients of somatoform disorder(s). Our study showed a significant prevalence of presence of various subtypes of somatoform disorders in broadly categorized and diagnosed patients of somatoform disorder(s).

\section{Bibliography}

1. American Psychiatric Association. Diagnostic and Statistical Manual of Mental Disorders. 4th ed. rev. Washington, D.C. American Psychiatric Association, 2000.

2. Schappert S.M. National Ambulatory Medical Care Survey: 1991 summary. Adv Data 1993; 230:1-16.

3. Kroenke K., Spitzer R.L., Williams J.B.W. The PHQ-15: validity of a new measure for evaluating the severity of somatic symptoms. Psychosom Med 2002; 64:258-66.

4. Fink P., Sorensen L., Engberg M., Holm M., Munk-Jorgensen P. Somatization in primary care. Prevalence, health care utilization, and general practitioner recognition. Psychosomatics. 1999; 40(4):330-38.

5. Pehlivanturk B., Unal F. Conversion disorder in children and adolescents; a 4year follow up study. J Psychosom Res 2002; 52:187-91.

6. Baruffol E., Thilmany M.C. Anxiety, depression, somatization and alcohol abuse. Prevalence rates in a general Belgian community sample. Acta Psychiatr Belg. 1993; 93(3):136-53.

7. McCahill M.E. Focus on the somataform disorders. Hospital Practice 1995; 30(2):59-66.
8. Stahl S.M. The psychopharmacology of painful physical symptoms in depression. $\mathbf{J}$ Clin Psychiatry. 2002; 63:5.

9. Mumford D.V., Bavington J.T., Bhatnagar K.S., Hussain Y., Mirza S., Naraghi M.M., et al. The Bradford Somatic Inventory. A multi-ethnic inventory of somatic symptoms reported by anxious and depressed patients in Britain and the IndoPakistan subcontinent. Br. J. Psychiatry; 1991:158:379-86.

10. World Health Organization. The ICD-10 classification of mental and behavioural disorders: diagnostic criteria for research. Geneva: World Health Organization; 1993.

11. Nakao M., Yamanaka G., Kuboki T. Suicidal ideation and somatic symptoms of patients with mind/body distress in a Japanese psychosomatic clinic. Suicide Life Threat Behav. 2002; 32(1):80-90. 